\title{
The Development of Quality Control System for the Embankment
}

\author{
Tomonori Takada* , Tatsunori Sada* , Syouzou Naemura** and Noriaki Aoyama** \\ * Mitsui Construction Co.,Ltd , Technical Research Institute , 518-1 Komaki , \\ Ngareyama- City, Chiba-Pref , 270-01 JAPAN \\ ** Ministry of Construction, Public Works Research Institute, 1 Asahi Tukuba-City, \\ Ibaragi-Pref , 305 JAPAN
}

\begin{abstract}
It is required for construction to have execution of high quality and high precision, however, Today's shortage of skilled workers and aging workingforce is causing a deterioration of quality. Therefore developing new technology for construction sites has become more important than ever before. And we need to promote the spread the new technology each construction sites.

To eliminate these problems, Ministry of Construction has been engrossed in new project which study about development of rationalized technology since 1990. Our development forms a part of the project in order to automation and rationalization on earthworks. In this report, we describe the results of joint research with Public Works Research Institute.
\end{abstract}

\section{Introduction}

With an aim to achieving the safe and efficient management of earthwork, landfill operation in particular, and thus streamlining and improving the efficiency of landfill operation, the authors developed a system that facilitates the automatic and real-time execution of management duties ( namely, quality control, work progress control and heavy equipment operation control). The system mounted on a tired roller, a typical type of compactors, is aimed to contribute to the achievement of speedy earthwork management by transmitting realtime control information to the operator and manager in charge.

Concrete development objectives include:

1) A tired roller onboard system that allows real-time and instantaneous positioning of a tired roller with enhanced accuracy and recording and displaying the roller 1 s travel route and operating conditions.

2) An onboard system that allows the evaluation of fill quality (the degree of compaction can be computed internally from density and moisture content measurements) at any time and location along the travel route. 
3) A system that measures and records the in-situ height of the roller to facilitate progress control of ongoing filling operations.

4) A system that integrates the above three functions to allow real-time transfer of data to the operator and manager in charge.

\section{Overview of the system}

\section{(1) Functions}

The system integrates [fill quality evaluation and work instruction function] to perform multiple-point or continuous evaluation of fill quality using a scattering-type radiation density and moisture gauge and to perform real-time measurement data processing, as well as to determine from statistical computations the planar distribution of the degrees of compaction of the filled ground to give the operator proper instructions. With the aid of GPS (Global Positioning System), the system also determines the position of a roller [positioning function] and records operational data [data recording]. In addition, the system is equipped with a function that helps acquire [height information] and grasp [the area covered by compaction] aimed to facilitate the progress control of filling operations.

\section{(2) System configuration}

The system consists of main system and three sets of subsystems. The main system is designed to output data and instructions on the monitor screen, monitor ongoing operations, record operational data and issue work instructions. The subsystem is intended to determine the position of heavy equipment, the degree of compaction and work progress. Figure 1 illustrates the system configuration. Shown in Figure 2 is a conceptual drawing of the system.

\section{(3) Basic specifications}

Given below are the primary specifications of the positioning system.

1) Measuring method: RTK (Real-time kinematic) - GPS

2) Devices used:

(a)GPS receiver: 4000SSE (fixed base), 4000SSE (movable base)

(b)Communication system: Specific low powered radio

(c)Communication distance: Line-of-sight 2-3 km (direct sight)

(d)Data processor: PC9801NC

3) Measurement range: Distance between the fixed and movable bases: $\max 2000 \mathrm{~m}$

4) Measurement accuracy: $10 \mathrm{~mm}$ (Target value on $X, Y$ coordinates)

$20 \mathrm{~mm}$ (Target value on $\mathrm{Z}$ coordinate)

5) Measurement time: 1-3 sec. (optionally selectable)

Given below are the primary specifications of the compaction measuring system.

1) Measuring method: Density: Gamma ray back scattering-type density and moisture gauge

2) Measurement range: Density: $1.2-2.5 \mathrm{~g} / \mathrm{cm} 3$, Moisture: $0-0.7 \mathrm{~g} / \mathrm{cm} 3$

3) Measurement accuracy: $2-5 \%$ of density (depends on measurement conditions)

4) Measurement time: 1 - $999 \mathrm{sec}$. (optionally selectable)

5) Display data: Date, measurement time, counting rate, No., measurement position

6) Data for storage: Date, measurement time, counting rate, measurement No., measurement position (from RTK-GPS)Stored into a hard disk or FDD. 
Main system

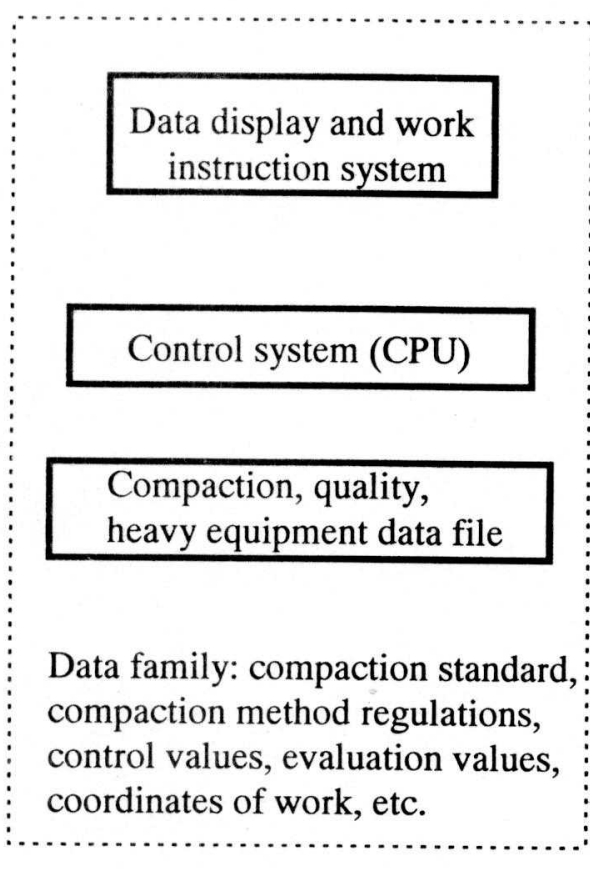

\section{Subsystem family}

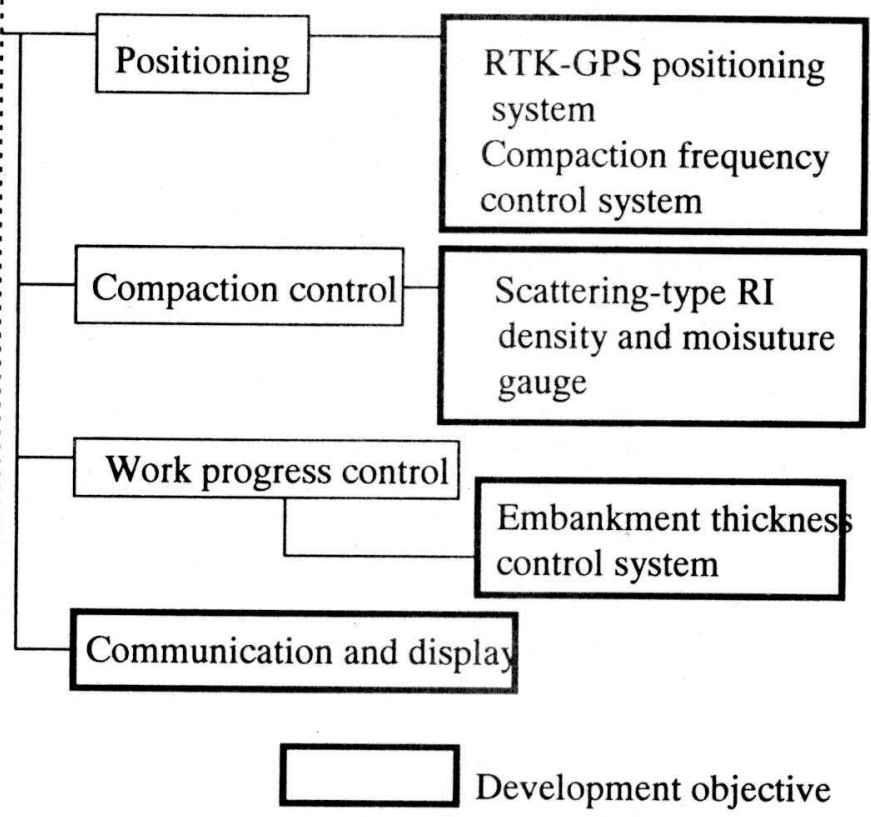

\section{Figure 1 The System Configuration}

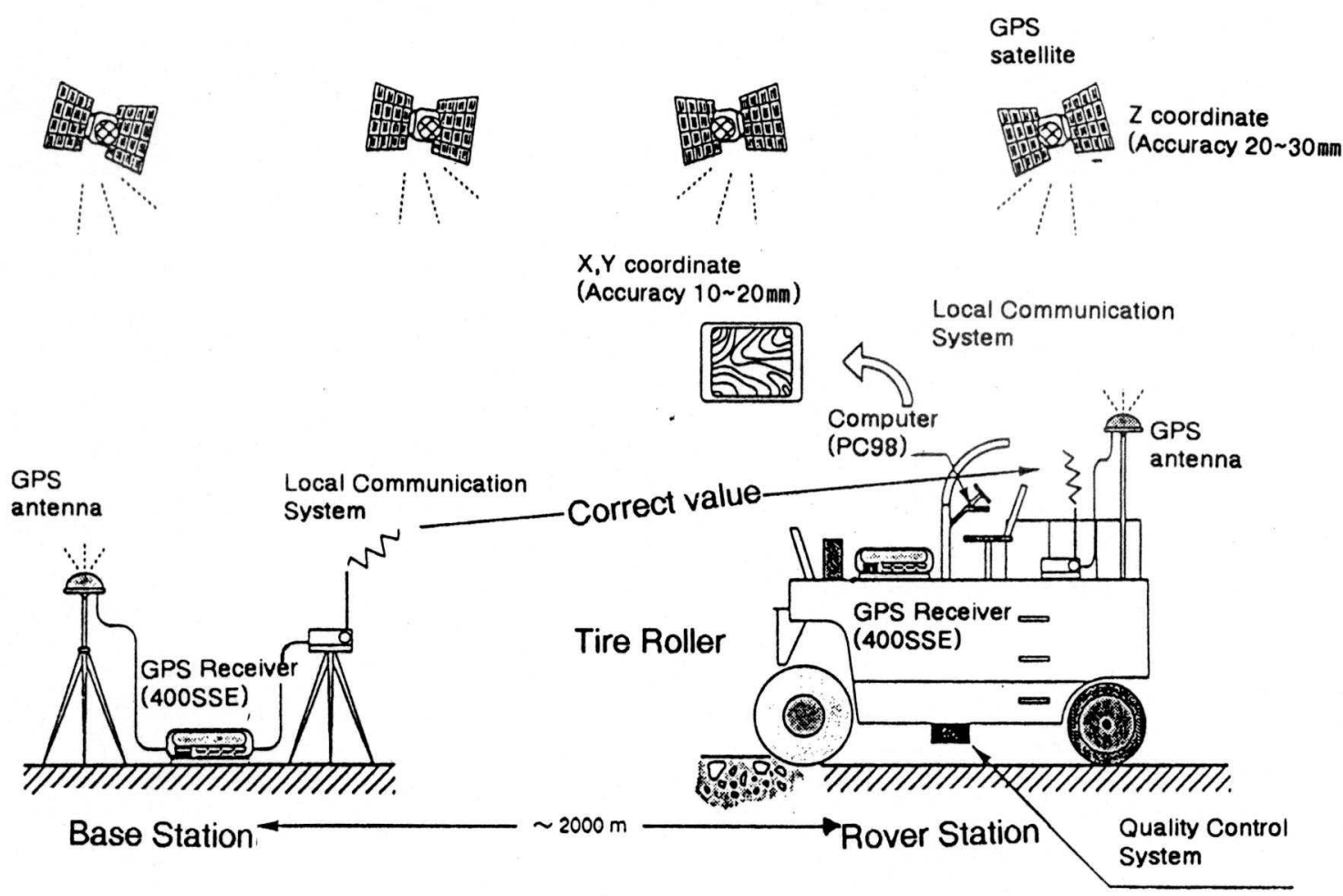

Figure 2 Conceptual Drawing of the System 


\section{Positioning system using RTK-GPS}

\section{(1) Configuration}

The system consists of, as is shown in Figure 2, GPS receiver and antenna, and communication system installed at the fixed base (known point on the coordinates) and movable base (measurement point). Dtata received at the fixed base is transmitted via the communicationsystem to the movable base, where the data is subjected to real-time processing and output to the monitor screen.

The communication system uses specific low powered radio readily available. The working distance is approximately $2 \mathrm{~km}$. The movable base uses a note-type personal computer for data display.

\section{(2) Functions}

1) Coordinate transformations

WGS-84 coordinates (latitude, longitude, ellipsoidal height) are transformed into Tokyo geodetic coordinates or plane rectangular coordinates ( $\mathrm{X}$ coordinate, $\mathrm{Y}$ coordinate, elevation).

2) Real-time display and recording of measurement data

The system displays and records plane rectangular coordinates ( $\mathrm{X}$ coordinate, $\mathrm{Y}$ coordinate, elevation) every one second in millimeters. A roller's instantaneous position and tracks can be monitored on a mesh that appears on the computer screen.

3) Guidance to the coordinates of a point

When the coordinates (X coordinate, $\mathrm{Y}$ coordinate) of any desired point (target point) are input, the system determines and outputs the position of the target point and a roller's instantaneous position on the mesh, along with the direction and distance to the target point .

\section{(3) Measurement accuracy}

The coordinates of points determined by electro-optical distance meter are compared with positioning data obtained with the system in Table 1 . The distances between the fixed base to the three positioning points range from $100 \mathrm{~m}$ to $300 \mathrm{~m}$. Errors were found to be within plus or minus $30 \mathrm{~mm}$ or so for the $\mathrm{X}$ coordinate, $\mathrm{Y}$ coordinate and height values.

Table 1 Result of Measurement accuracy

\begin{tabular}{|c|c|c|c|c|c|c|c|c|c|}
\hline \multirow[t]{2}{*}{ Point NO } & \multicolumn{2}{|r|}{$\mathrm{X}$ coordinates } & & \multicolumn{2}{|c|}{ Y coordinates } & \multicolumn{4}{|c|}{ Hight coordinates } \\
\hline & correctness & RTK-GPS & Difference & correctness & RTK-GPS & Difference & correctness & \begin{tabular}{l|} 
RTK-GPS \\
\end{tabular} & Difference \\
\hline 1 & 110003.220 & 110003.206 & 0.014 & 84545.912 & 84545.911 & $\begin{array}{r}0.001 \\
\end{array}$ & 91.596 & 91.629 & -0.033 \\
\hline 2 & 109913.919 & 109913.945 & -0.026 & 84635.841 & 84635.841 & 0.006 & 85.277 & 85.287 & -0.010 \\
\hline 3 & 110111.683 & 110111.673 & 0.010 & 84519.033 & 84519.048 & -0.015 & 79.913 & 79.932 & -0.019 \\
\hline
\end{tabular}

\section{Compaction measuring system by scattering-type RI gauge}

\section{(1) Mechanism of RI measurement}

RI measurement is conceived as a method to determine subsoil density and moisture content. It utilizes the interaction between radioactive rays emitted from a radioisotope and the constituent elements (atoms) of soil. Density measurement uses gamma rays as radioactive rays and utilizes the soil density-dependency inherent in the interaction. Meanwhile moisture 
content measurement uses neutrons instead of gamma rays. It utilizes the reaction induced by a collision of hydrogen atoms contained in soil water with fast neutrons, where fast neutrons are transformed into thermal neutrons.

The basic concept of IR measurement is to detect exclusively radioactive rays that interact with soil constituent atoms.

In Figure 3, an overview of the mechanism of this method is presented.

\section{(2) Overview of the measuring instrument}

The design of the measuring instrument used is twofold: separate-type and integral-type. The former consists of separate components of radiation source, detector, and display.

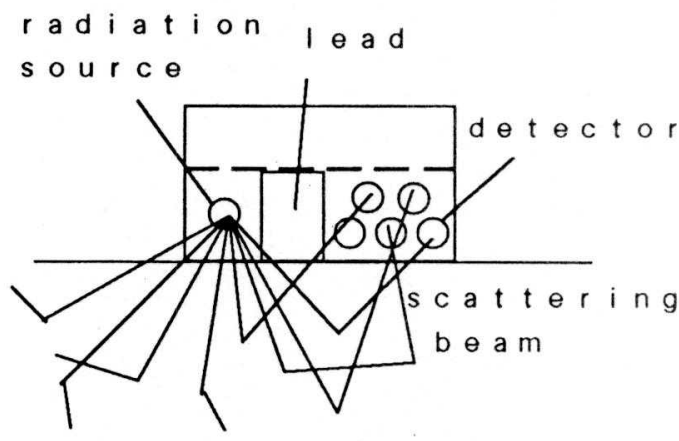

Figure 3 Outline of RI Scattering Gauge The latter is a compact version of the former, in which these separate components are integrated in a single unit. The separate-type adopts various new ideas are inventions, radiation screening for example, to attain shortest possible density and moisture content measurement time. Although continuous measurement at one second intervals is possible, the system is heavy in weight.

Meanwhile in the case of the handy and lighter integral-type, manual measurement is possible, however, the measurement time is fixed at 60 seconds, as in the case of commonly used transmission type. The compaction measuring system uses the separate-type to carry out continuous measurement $20 \mathrm{~mm}$ above the ground surface. The system consists of main system and data processor.

\section{(3) Characteristics}

The measurement method eliminates the need for holes for the insertion of a cylindrical container containing radiation source, rendering itself purely nondestructive. The basic concept of the compaction measuring system is that a scattering-type RI density and moisture gauge loaded tired-roller carries out as it travels non-contact and nondestructive density/moisture content measurement over the embankment surface. It then computes the degree of compaction and air content to determine the quality of the filling operation immediately after completion, the results of which are fed back without delay to the operator. The system does not require any kind of permission or qualifications and is available at any site.A special calibration technique employed makes density/moisture measurement $10-20$ $\mathrm{mm}$ above the ground surface possible (Photograph 1).

Measurement data can be obtained once every second, enabling the planar measurement over the length and breadth of a measurement target.

And compaction measurement data obtained at individual measurement points (the coordinates of such points are determined by the positioning system using RTK-GPS) is output in the form of a compaction map that appears on the display installed in front of the operator's seat. From the real-ime data, the operator can grasp the state of ongoing operations including zones of defective compaction. Moreover,positioning and compaction data is continuously stored in a floppy disk or a hard disk throughout the iterated compaction process. The stored data will be eventually used as execution control data. 


\section{Display and communication system}

The configuration of the display and communication system is presented in Figure 4.

Given below are output data.

1) Dry density, compaction, moisture content measurements.

2) Zonal distribution of compaction measurements.

3) Position of a travelling roller.

4) A roller's travel route.

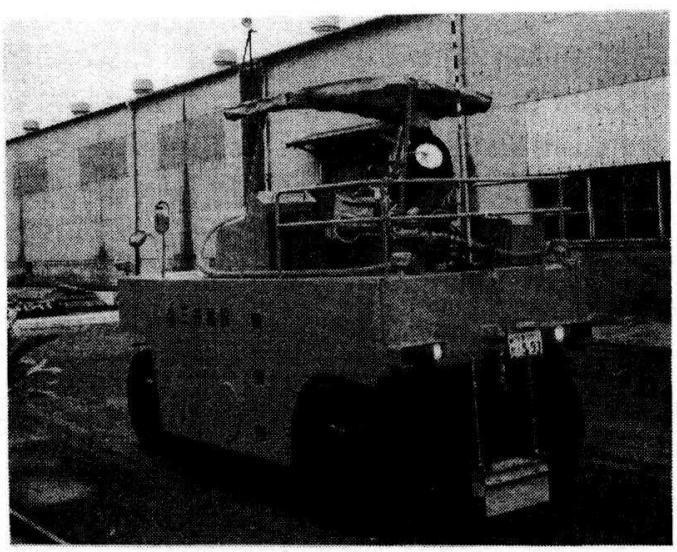

Photograph 1 The measuring instrument

\section{Concluding remarks}

The satisfactory results obtained from the introduction of the system can be summarized as follows:

1) With the use of RTK-GPS, the operator can monitor the instantaneous position $(\mathrm{X}, \mathrm{Y}, \mathrm{Z}$ coordinate) and travel speed of his roller and the number of compaction to determine the adequacy of ongoing op-erations.

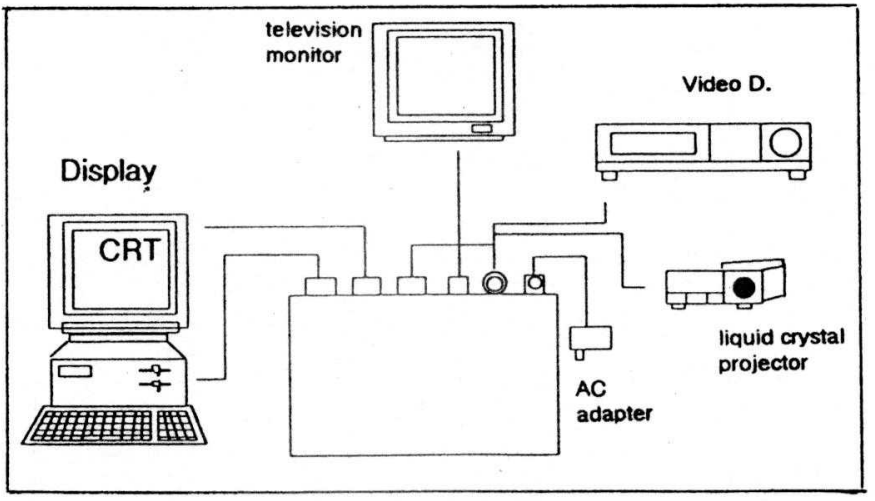

Figure 4 Basic construction of Display system

2) With the use of the scattering-type RI measurement technique (the product of collaborative research and development by the Ministry of Construction and the private sector), the compaction quality of an embankment can be evaluated continuously throughout the rolling operation. The evaluation results are presented visually to the operator or a manager in charge.

3) When the finishing phase is reached, the system outputs information containing particular sections requiring further compaction, along with points of a tention and consideration for subsequent operations. The evaluation of the amount of work completed is also possible.

4) Real-time presentation and recording of data obtained from the evaluation of the quality of a finished product, the amount of work completed, and the rate of heavy equipment operation is possible. The data can be reflected in various control services involved, such as progress, quality and prime cost.

5) The use of the communication system enables the presentation of measured data, wherever necessary, to not only the operator but a manager in charge.

Problems requiring further review include:

1) Applicability of the system to a wide range of soil variation.

2) Study of measurement techniques that best fit specific site conditions (construction equipment, soil type, scale of work, etc.).

3) Real-time processing of measurement data for construction control purposes, and further review of the feedback technique. 